\title{
Minimum volume stability limits for axisymmetric liquid bridges subject to steady axial acceleration
}

\author{
J.M. Perales, J. Meseguer \\ Laboratorio de Aerodinámica, ETSI Aeronáuticos, Universidad Politécnica, 28040 Madrid, Spain
}

and

I. Martínez

ETSI Navales, Universidad Politécnica, 28040 Madrid, Spain

\begin{abstract}
In this paper the influence of an axial microgravity on the minimum volume stability limit of axisymmetric liquid bridges between unequal disks is analyzed both theoretically and experimentally. The results here presented extend the knowledge of the static behaviour of liquid bridges to fluid configurations different from those studied up to now (almost equal disks). Experimental results, obtained by simulating microgravity conditions by the neutral buoyancy technique, are also presented and are shown to be in complete agreement with theoretical ones.
\end{abstract}

\section{Introduction}

A liquid bridge is an idealization of the fluid configuration appearing in the crystal growth technique known as floating zone melting. The liquid bridge configuration, as sketched in fig. 1, consists of a mass of liquid held by surface tension forces between two parallel, coaxial, solid disks. Such a fluid configuration can be identified by the following dimensionless parameters: the slenderness, $A=L / 2 R_{0}$, where $L$ is the distance between the disks and $R_{0}=\left(R_{1}+R_{2}\right) / 2$ the mean radius, which is used as characteristic length; the ratio of the radius of the smaller disk to the radius of the larger one, $K=R_{1} / R_{2}$; the dimensionless volume of liquid, $V=V / R_{0}^{3}, V$ being the physical volume; and the Bond number, $B=\Delta \rho g R_{0}^{2} / \sigma$, where $\Delta \rho$ is the difference between the liquid bridge density and the surrounding medium density (either a gas or another liquid), $g$ the axial acceleration and $\sigma$ the surface tension.
The availability of flight opportunities to carry out fluid science experiments in microgravity is given rise to interest in the study of the behaviour of liquid masses under microgravity conditions, the liquid bridge being one of the fluid configurations which is receiving increasing attention in those experiments [1-8]. The mechanical aspects of the liquid bridge problem have received the attention of many investigators during the last two decades and, leaving apart dynamic effects, one can find in the literature a large number of papers dealing with the equilibrium shapes and the static stability limits of liquid bridges. It is well known that for each set of dimensionless parameters $(\Lambda$, $K, B)$, there is a minimum volume of liquid for which a stable liquid bridge can be formed. Stability limits of minimum volume of liquid bridges at rest between equal disks ( $K=1)$ under zero gravity conditions $(B=0)$ have been studied in refs. [9-14], among others; the influence of microgravity $(B \neq 0)$ has been considered, for instance, in 


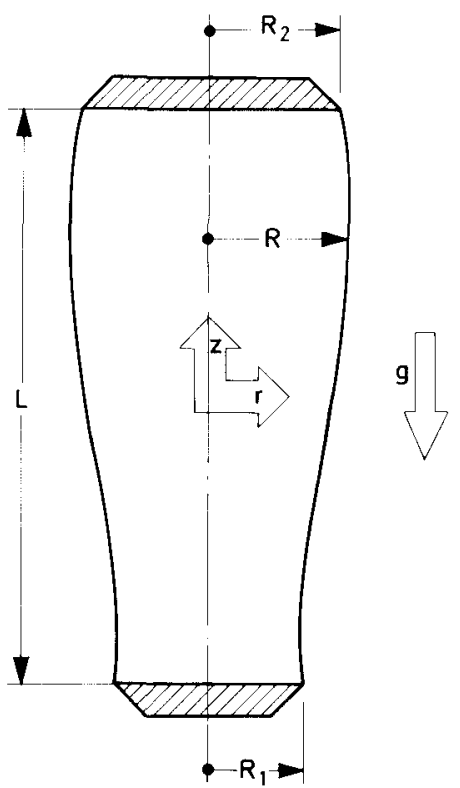

Fig. 1. Geometry and coordinate systems for the liquid bridge problem.

refs. [15-17] in the case of liquid bridges between equal disks $(K=1)$, whereas the influence on stability limits of different disks $(K \neq 1)$ in zero gravity conditions $(B=0)$ has been analyzed in refs. [17-19]. Both, Bond number and unequal disks, are non-symmetric effects. Each one separately decreases the stability of the liquid column (the volume of liquid must be increased to keep the configuration stable), but both effects combined may cancel each other or, in other words, each of these effects can be a stabilizer for the remaining one. This behaviour has been analyzed in refs. [20,21], although published results concern only liquid bridges between almost equal disks $(K \sim 1)$ and very low values of the Bond number $(B \sim 0)$.

This paper tries to fill the gap existing in the knowledge on the stability limits for minimum volume of liquid bridges between unequal disks in an axial microgravity field $(K \neq 1, B \neq 0)$. Those limits have been calculated for a wide range of the parameters $K$ and $B(0.5 \leq K \leq 1,|B| \leq 0.5)$ and some experiments have been performed in order to verify theoretical predictions.

From the experimental point of view, the studies of liquid bridges carried out in earth-based laboratories are strongly constricted because the maximum stable length of a liquid bridge is of the order of a few milimetres. Slender liquid bridges can only be obtained by working on a very small scale, or by simulating microgravity conditions by using the neutral buoyancy technique, with one liquid surrounded by a second one with which it is immiscible and of precisely the same density. Neutral buoyancy has been extensively used [22$35]$ because this technique is very appropriate for hydrostatic studies. Experiments have been performed on liquid bridge configurations with $K=1$ and $K=0.82$, and different values of the Bond number $B$ ranging from 0.5 to -0.5 , experimental results being in agreement with analytical ones.

\section{Theoretical analysis}

In the following, unless otherwise stated, all lengths are made dimensionless with $R_{0}$. Axisymmetric equilibrium shapes of liquid bridges anchored to the sharp edges of the supporting discs are characterized by the dimensionless meridian curve (outer shape) $R=R(z$ ), which is a function of the configuration and stimuli applied, namely, the ratio of the radius of the supporting discs $K$, slenderness $\Lambda$, liquid volume $V$, residual acceleration in the axial direction of the column measured by the Bond number $B$, and solid-body rotation speed, although this last effect is not considered in the following. Then, assuming the set of parameters $(K, \Lambda, V, B)$ given, the outer shape, $R=R(z)$ can be computed by numerical integration of the second order differential (Young-Laplace) equation:

$C(z)-B z+P=0$,

where $C(z)$ is the local mean curvature at a point in the shape:

$C(z)=\frac{R_{z z}}{\left(1+R_{z}^{2}\right)^{3 / 2}}-\frac{1}{R\left(1+R_{z}^{2}\right)^{1 / 2}}$,

and $P$ is the non-dimensional gauge pressure at 
the origin, an implicit constant that must be also computed from the boundary conditions:

$R(-\Lambda)=2 K /(1+K), \quad R(+\Lambda)=2 /(1+K)$,

$\int_{-\Lambda}^{\Lambda} \pi R^{2}(z) \mathrm{d} z=V$

This is a typical boundary-value problem that has been solved here as an initial-value problem by the shooting method as explained below.

The procedure to find stability limits of minimum volume has been as follows: a clearly stable point in the parameter set $(K, A, V, B)$ is chosen (for instance, a short nearly cylindrical liquid bridge configuration), and subsequent points in the set $(K, A, V, B)$ are found maintaining constant all except $V$, which is decreased a certain amount at every step.

To start with, finding the shape corresponding to a given set $(K, \Lambda, V, B)$ is not so easy; with a shooting (Runge-Kutta) routine one finds the solution for the set $(T, A, P, B)$, where $T$ is the slope at $z=-\Lambda$ of the meridian curve and $P$ is the internal parameter that appears in eq. (1). A pair of corresponding values $K, V$ is obtained, but not the desired one.

A second iterative loop (the first one is for the Runge-Kutta) uses a Newton-Raphson algorithm to solve for the system of equations $K(T, P)=K$ and $V(T, P)=V$. The problem with this algorithm is that it only works if the initial guess for $T$ and $P$ is close enough to the solution. Fortunately, if the shape to be found is very stable, the procedure converges quickly. When one is approaching the stability limit, if the jumps in $V$ are small, the values $T$ and $P$ will vary little from shape to shape and the initial guess becomes very good. Besides the shape, the Newton-Raphson routine gives also the Jacobian of the transformation, $J$,

$J=\left|\begin{array}{ll}\frac{\partial K}{\partial T} & \frac{\partial V}{\partial T} \\ \frac{\partial K}{\partial P} & \frac{\partial V}{\partial P}\end{array}\right|$,
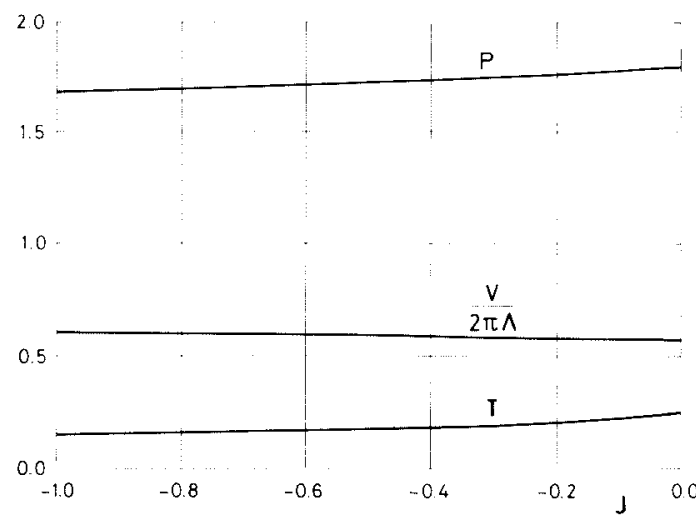

Fig. 2. Variation with the Jacobian, $J$, of: (a) the slope at $z=-\Lambda$ of the liquid bridge shape, $T(J)$; (b) the reference pressure, $P(J)$; (c) the ratio of the dimensinless volume of liquid to the volume of a cylinder of the same slenderness, $V(J) / 2 \pi \Lambda$. The results correspond to a liquid bridge with $\Lambda=2, K=0.7$ and $B=0.2$.

that approaches zero as the stability limit is approached.

How close a given meridian curve $R=$ $R(z, K, \Lambda, V, B)$ is to an equilibrium shape, can be measured by the deviation $\epsilon$, defined as:

$\epsilon=\int_{-\Lambda}^{\Lambda}[C(z)-B z+P]^{2} \mathrm{~d} z$.

A typical value of $\epsilon$ used in the calculations could be $\epsilon=10^{-5}$.

The stability limit is found by extrapolation to $J=0$ of the functions $T(J)$ and $P(J)$, giving the limiting shape and in particular the minimum volume, which was the aim of this exercise; an example of the functions $T(J), P(J)$ and $V(J)$ is plotted in fig. 2.

Some of the results obtained are shown in fig. 3 , where the influence of the Bond number on the minimum volume stability limit for different values of the parameter $K$ can be observed. A liquid bridge configuration, represented by a point in the $\Lambda-V$ diagram, will be stable if the point lies above the corresponding stability curve (identified by the values of $K$ and $B$ ); otherwise the configuration will be unstable.

The results show an aspect of the static behaviour of liquid bridges between unequal disks already pointed out in refs. [20-21]: for given values of $\Lambda$ and $K(K \neq 1)$, the Bond number 

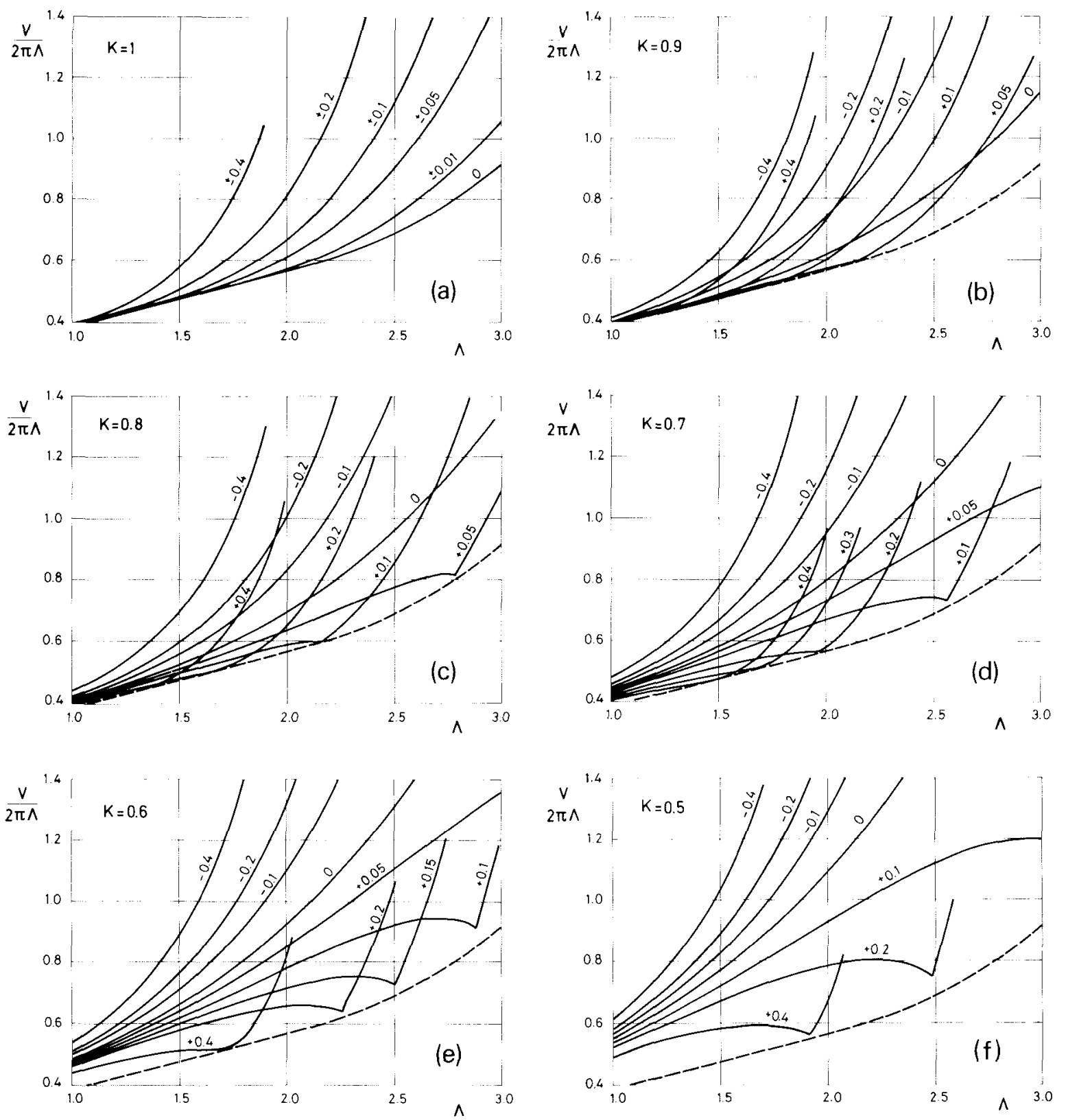

Fig. 3. Variation with the slenderness, $\Lambda$, of the minimum volume stability limit divided by the volume of a cylinder of the same slenderness $V / 2 \pi \Lambda$. Numbers on the curves indicate the value of the Bond number, $B$. In the plots corresponding to $K \neq 1$, the dashed line indicates the limit $K=1, B=0$.

may increase the stability of liquid bridges (the minimum volume may be smaller than that of the stability limit corresponding to $B=0$ and the resulting configuration be still stable) and this is because the unequality of the disks is a non-sym- metric effect like the Bond number, so that both effects can be added (in that case the values of the minimum volume stability limit increase) or can cancel one another. Observe that if both disks are almost equal in diameter $(0.8 \leq K \leq 1)$, the differ- 
ent stability-limit curves have an envelope which roughly coincides with the stability limit corresponding to $K=1, \quad B=0$, which could be regarded as an absolute minimum volume stability limit, as already stated in ref. [21]. The envelope does not coincide with the limit for $K=1, B=0$ when both disks are clearly different, and as $K$ decreases the absolute minimum volume stability limit increases.

\section{Experimental technique and results}

Experiments have been performed in an apparatus called Tele-Operated Plateau Tank (TOPT) Facility already described in ref. [36]. The working configuration consists of a column of silicone oil (with kinematic viscosity $20 \times 10^{-6} \mathrm{~m}^{2}$ $\mathrm{s}^{-1}$ ) placed vertically inside a mixture of methanol and water of almost the same density of the silicone oil. The overall arrangement, as sketched in fig. 4, consists of a tank which contains the methanol-water mixture and the liquid bridge held between two coaxial disks. Both disks are made of Perspex, in the shape of a frustrum cone, to provide sharp edges, their diameter being $30 \mathrm{~mm}$ for the experiments with $K=1$, and 30 and $24.5 \mathrm{~mm}$ in the case $K=0.82$. The injection and removal of the working fluid is done through a $4 \mathrm{~mm}$ diameter hole in the centre of the upper disk. The working surface of the bottom disk is flat, whereas that of the feeding disk is slightly conic, the purpose of which is to facilitate the evacuation of air

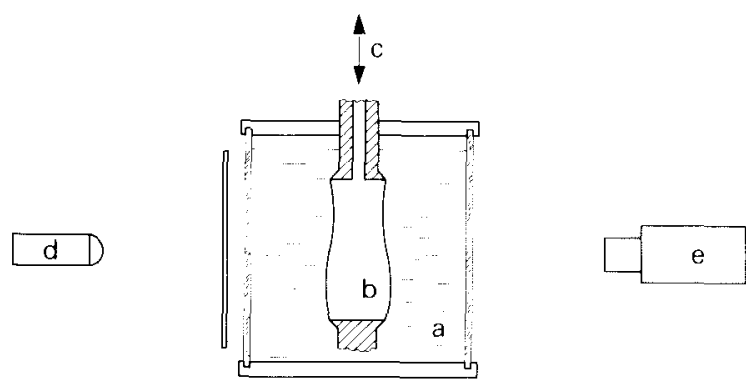

Fig. 4. Sketch of the experimental facility: (a) plateau tank; (b) liquid bridge; (c) liquid injection and removal; (d) background illumination; (e) TV camera. bubbles trapped in the liquid bridge through the injection hole. Working fluid injection and removal is done with a calibrated syringe, the piston of which is driven by a variable speed electric motor. Liquid displaced by the piston passes through the filling duct. The filling duct has a three-way valve with a purge duct which traps air bubbles coming from the upper disk.

Background illumination consists of a $150 \mathrm{~W}$ fiber optic illuminator (similar to those used in microscopy). A CCD video camera, placed $1 \mathrm{~m}$ away, is used for image recording. To enhance the visualization of shape of the liquid bridge (outline of its free-surface), only half of the background illumination plane is illuminated. Automated image analysis has been developed, so that the liquid contour can be extracted in near real time from the video frames [36].

The development of a typical experimental sequence is as follows: for a given value of $K$ (to change the value of $K$ requires to change some of the supporting disks), a value of the Bond number is fixed by selecting the appropriate density of the water-methanol mixture. Thus a liquid bridge of the desired slenderness and volume is established From now on the process is quite similar to that described in ref. [29], the major difference being that now all the process and the data analyses are automatically made through a computer. Once the liquid bridge is formed, the experiment consists of sucking liquid from the bridge at constant slenderness until the liquid bridge disrupts. This is performed by a slow withdrawal in steps of $0.5 \mathrm{~cm}^{3}$, separated by a $20 \mathrm{~s}$ wait to allow for instabilities, if any, to develop. The process stops when the liquid bridge disruption takes place. Then a new liquid bridge of another slenderness is formed and a new run starts. Recorded information during each test is, in addition ot the geometrical parameters (disks separation, syringe piston position), a set of liquid-bridge interface shapes which are used further to calculate both the Bond number and the minimum volume for which the liquid bridge is stable.

Once all the tests for a given value of $B$ are performed, the value of the Bond number is modified by adding alcohol or water to the bath, and a new experimental sequence starts. 
One of the problems that could arise when a methanol-water mixture is used as surrounding liquid is the existence of a density gradient along the tank. This phenomenon was experimentally studied by Tagg et al. [27] and a density gradient as high as $50 \mathrm{~kg} \mathrm{~m}^{-4}$ was found with their experimental configuration, although these authors did not state whether the density gradient was due to inhomogeneities in the mixture or to the significant vertical temperature gradient existing in their experiment.

Since we were aware of this problem, a previous experiment was performed aiming at measure density gradients in the bath. For this purpose a moiré deflectometer was used. Results from this previous experiment show that density gradients inside the bath are negligible except in a thin layer close to its free surface.

A second problem to be taken into account when alcohol-water mixtures are used is that alcohol evaporates rather quickly, and then the bath density varies with time, which implies that the Bond number also varies with time. To minimize alcohol evaporation, the tank must be kept as tight as possible.

The value of the Bond number has been measured by fitting theoretical interface shapes to experimental ones. These measurements show that the Bond number varies with time along the different experimental sequences performed, the rate of change of the bath density being of the order of $7 \times 10^{-5} \mathrm{~kg} \mathrm{~m}^{-3} \mathrm{~s}^{-1}$. This means that within one experimental sequence (a typical value of the time spent to perform an experimental sequence could be some $60 \mathrm{~min}$ ), the bath density changes with $0.25 \mathrm{~kg} \mathrm{~m}^{-3}$, which gives Bond number differences of the order of 0.025 between the start and the end of the experimental sequence. However, this is not the reference value for the Bond number variation for a comparison between experimental and theoretical results. In effect, minimum volume stability limits are less sensitive to Bond number when the slenderness of the liquid bridge decreases. This is shown in fig. 5, where some experimental results are compared with the theoretical ones corresponding to $B=0.015$ and $B=0.025$. As one can observe, a Bond number difference of 0.01 gives differences in the values of

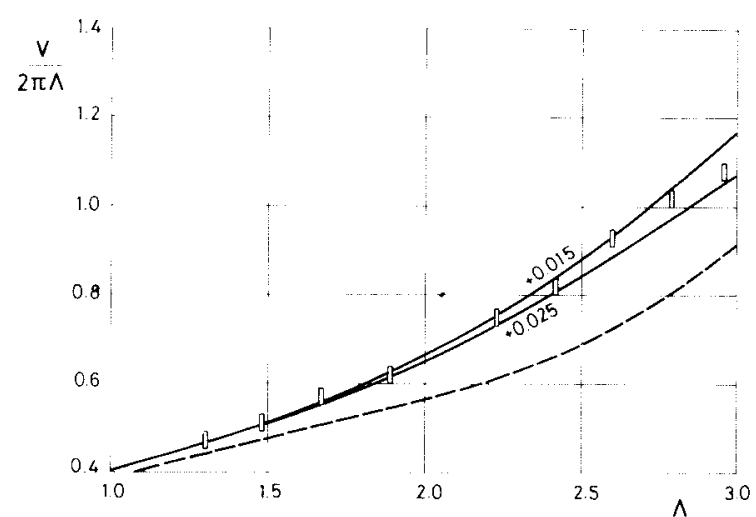

Fig. 5. Influence of the Bond number, $B$, on the minimum volume stability limit divided by the volume of a cylinder of the same slenderness, $V / 2 \pi \Lambda$. The symbols indicate experimental results, whereas the solid lines correspond to theoretical ones. The number on the curves indicates the value of $B$. The results correspond to liquid bridges between unequal disks ( $K=0.82$ ). The dashed line indicates the limit $K=1, B=0$.

the volume stability limits which are less than the error in the experimental volume measurements for liquid bridges with $\Lambda \leq 2.5$. This seems to indicate that the Bond number variation is only significant with $\Lambda>2.5$. However, the time needed to perform the experiments with $\Lambda>2.5$ was approximately $1 / 3$ of the time spent in the whole sequence. Thus, the reference value for the Bond

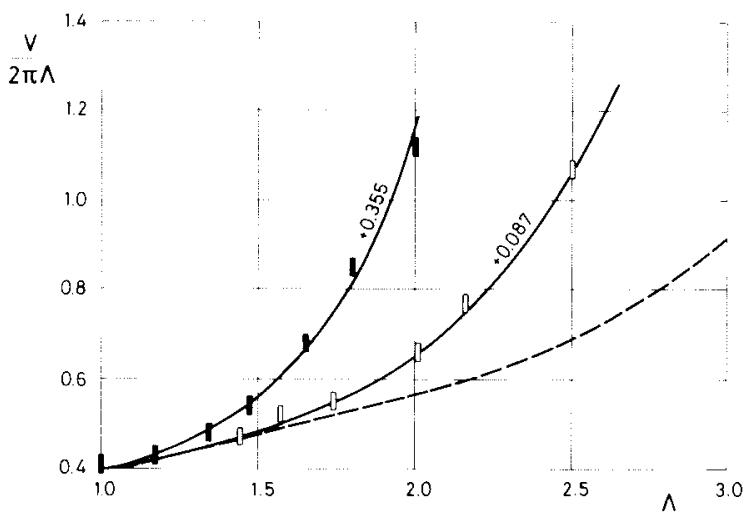

Fig. 6. Variation with the Bond number, $B$, of the minimum volume stability limit divided by the volume of a cylinder of the same slenderness, $V / 2 \pi \Lambda$. The symbols indicate experimental results, whereas the solid lines correspond to theoretical ones. The number on the curves indicates the value of $B$. The results correspond to liquid bridges between equal disks ( $K=$ 1). The dashed line indicates the limit corresponding to $B=0$. 


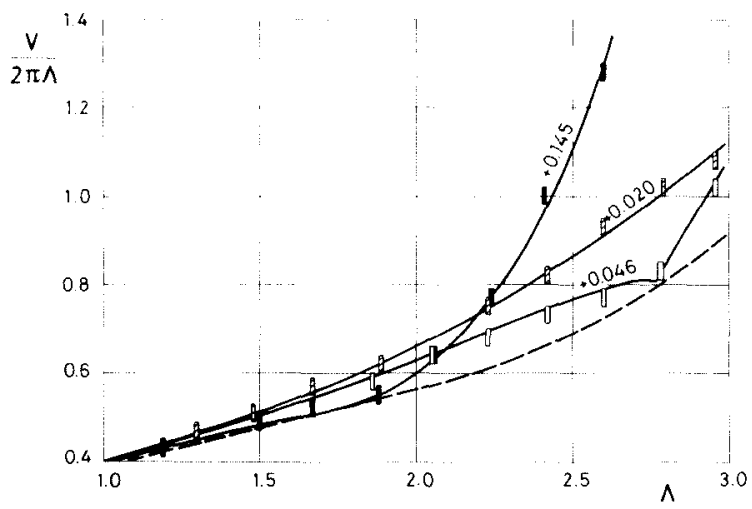

Fig. 7. Variation with the Bond number, $B$, of the minimum volume stability limit divided by the volume of a cylinder of the same slenderness, $V / 2 \pi \Lambda$. The symbols indicate experimental results, whereas the solid lines correspond to theoretical ones. The number on the curves indicates the value of $B$. The results correspond to liquid bridges between unequal disks ( $K=0.82$ ). The dashed line indicates the limit $K=1, B=0$.

number variation with time should be at least three times smaller than that quoted above.

Experimental results are shown in fig. $6(K=1)$ and in fig. $7(K=0.82)$. Experimental results show the same trends as theoretical ones. When both disks are equal in diameter, the minimum volume stability limit increases as the Bond number increases, but when $K \neq 1$ ( $K=0.82)$, the behaviour of the liquid bridge is rather different and, depending on the value of the slenderness, the Bond number can be a stabilizing effect, as clearly shown by the results presented in fig. 7 .

\section{Acknowledgement}

This work has been supported by the Spanish Comisión Interministerial de Ciencia y Tecnología (CICYT, Project No. ESP 88-0359).

\section{References}

[1] 1. Martínez, in: Material Sciences under Microgravity, ESA SP-222 (ESA, Paris, 1984).

[2] I. Martínez and A. Eyer, J. Crystal Growth 75 (1986) 535.

[3] I. Martinez and A. Sanz, ESA J. 9 (1985) 323.

[4] J. Meseguer, A. Sanz and J. López, J. Crystal Growth 78 (1986) 325 .
[5] I. Martínez, Acta Astronautica 15 (1987) 449.

[6] I. Martínez, in: Material Sciences under Microgravity, ESA SP-256 (ESA, Paris, 1987).

[7] 1. Martinez and J. Meseguer, in: Scientific Results of the German Spacelab Mission D1, Eds. P.R. Sahm, R. Jansen and M.H. Keller (DFVLR, Köln, 1987).

[8] I. Martínez, A. Sanz, J.M. Perales and J. Meseguer, ESA J. 12 (1988) 483

[9] J.M. Haynes, J. Colloid Interface Sci. 32 (1970) 652.

[10] M.A. Erle, R.D. Gillette and D.C. Dyson, Chem. Eng. J. 1 (1970) 97.

[11] R.D. Gillette and D.C. Dyson, Chem. Eng. J. 2 (1971) 44

[12] I. Martínez, in: Material Sciences in Space, ESA SP-114 (ESA, Paris, 1976).

[13] I. Da Riva and I. Martínez, in: Material Sciences in Space, ESA SP-142 (ESA, Paris, 1979).

[14] 1. Martínez, in: Materials Sciences under Microgravity, ESA SP-191 (ESA, Paris, 1983).

[15] S.R. Coriell and M.R. Cordes, J. Crystal Growth 42 (1977) 466.

[16] J.M. Vega and J.M. Perales, in: Materials Sciences under Microgravity, ESA SP-191 (ESA, Paris, 1983).

[17] I. Martínez, J.M. Haynes and D. Langbein, in: Fluid Sciences and Materials Science in Space, Ed. H.U. Walter (Springer, Berlin, 1987).

[18] I. Martinez and J.M. Perales, J. Crystal Growth 78 (1986) 369.

[19] J. Meseguer, J. Crystal Growth 67 (1984) 141.

[20] J. Meseguer, J. Crystal Growth 73 (1985) 599.

[21] J. Meseguer, A. Sanz and J.M. Perales, Appl. Microgravity Tech. 2 (1990) 186.

[22] G.C. Mason, J. Colloid Interface Sci. 32 (1970) 172.

[23] J.R. Carruthers and M. Grasso, J. Appl. Phys. 43 (1972) 436.

[24] J.R. Carruthers and M. Grasso, J. Crystal Growth 13/14 (1972) 611.

[25] S.R. Coriell, S.C. Hardy and M.R. Cordes, J. Colloid Interface Sci. 60 (1977) 126.

[26] H. Rodot, C. Bisch and A. Lasek, Acta Astronautica 6 (1979) 1083

[27] R. Tagg, L. Cammarck, A. Croonquist and T.G. Wang, Report JPL 900-954 (JPL, Pasadena, 1980).

[28] A. Sanz and I. Martínez, J. Colloid Interface Sci. 93 (1983) 235.

[29] J. Meseguer and A. Sanz, J. Fluid Mech. 153 (1985) 83.

[30] A. Sanz, J. Fluid Mech. 156 (1985) 101.

[31] J. Meseguer, L.A. Mayo, J.C. Llorente and A. Fernández, J. Crystal Growth 73 (1985) 609.

[32] M.J. Russo and P.H. Steen, J. Colloid Interface Sci. 113 (1986) 154.

[33] A. Sanz and J. López-Díez, J. Fluid Mech. 205 (1989) 503.

[34] A. Sanz and J.M. Perales, Appl. Microgravity Tech. 2 (1989) 133

[35] H. González F.M.J. McCluskey, A. Castellanos and A. Barrero, J. Fluid Mech. 206 (1989) 545.

[36] A. Sanz-Andrés, J.M. Perales, P. Rodríguez de Francisco and A. Sanz-Lobera, in: Materials Sciences under Microgravity, ESA SP-295 (ESA, Paris, 1990). 